\title{
Fosfomycin susceptibility of uropathogens at Charlotte Maxeke Johannesburg Academic Hospital
}

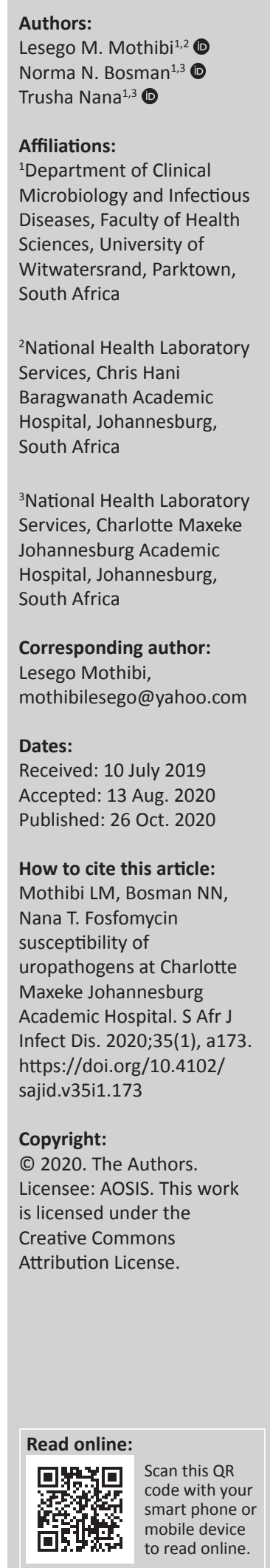

Background: Multidrug-resistant uropathogens are becoming widespread both in community and hospital setting. Safe yet effective treatments are a priority. Fosfomycin is an antibacterial that displays good activity against most bacteria causing urinary tract infections (UTIs), including multidrug-resistant bacteria. The aim of this study was to evaluate fosfomycin susceptibility for uropathogens isolated from a microbiology laboratory at a tertiary academic hospital. In addition, this was compared to the susceptibility of other oral antimicrobials.

Methods: We conducted a retrospective analysis of laboratory reports for uropathogens isolated at Charlotte Maxeke Johannesburg Academic Hospital from September 2015 to August 2017. Antimicrobial susceptibility testing of the isolates was performed using the Kirby-Bauer disk diffusion method or the $\operatorname{Vitek}^{\oplus} 2$ system according to the Clinical and Laboratory Standards Institute.

Results: Overall susceptibility of fosfomycin for the 4700 Enterobacteriaceae isolates was 95.7\%; 95\% confidence interval (CI) 95.1-96.2. The overall susceptibility for fosfomycin against the gram-positives was $98.6 \%$. There were $37.9 \%$ multidrug-resistant Enterobacteriaceae (MDRE) isolated during the study period. Fosfomycin displayed activity against $94.4 \%$ of extended-spectrum $\beta$-lactamase (ESBL) producers and $90.7 \%$ for carbapenem-resistant Enterobacteriaceae (CRE). None of the methicillin-resistant Staphylococcus aureus and vancomycin-resistant Enterococcus isolates tested was fosfomycin resistant. The overall in vitro susceptibility was significantly higher for fosfomycin $(p=0.0001)$ compared to amoxicillin/ clavulanic acid, cephalexin, cefuroxime, ciprofloxacin, trimethoprim/sulfamethoxazole and nitrofurantoin.

Conclusion: This study confirmed the high susceptibility of fosfomycin against UTI pathogens isolated at our institution. In an era of increasing antimicrobial resistance, fosfomycin represents a potential option for the treatment of UTIs at Charlotte Maxeke Johannesburg Academic Hospital.

Keywords: fosfomycin susceptibility; urinary tract infections; uropathogens; multidrugresistant organisms; ciprofloxacin resistance; hospital; ambulatory.

\section{Introduction}

Urinary tract infections (UTIs) have become the most common bacterial infections, equally found in the community and in hospitals. ${ }^{1}$ Antibiotics such as ciprofloxacin, trimethoprim/ sulfamethoxazole and nitrofurantoin were used successfully as therapy for UTIs. ${ }^{2}$ However, because of the increased drug resistance to these antibiotics, the choice of empiric antimicrobials for UTIs has become more challenging. ${ }^{3,4}$ As a result, there is a growing need to find novel treatment options or to re-examine old antibiotics such as fosfomycin.

Fosfomycin is a broad-spectrum antimicrobial agent that inhibits the synthesis of the bacterial cell wall of both gram-negative and gram-positive bacteria. ${ }^{5}$ The Infectious Diseases Society of America (IDSA) 2010 guidelines on the treatment of uncomplicated UTIs recommend fosfomycin $3 \mathrm{~g}$ oral single-dose as one of the first-line treatment options. ${ }^{6}$ Fosfomycin displays good activity against most bacteria-causing UTIs, including multidrug-resistant (MDR) organisms. ${ }^{5}$ It is well tolerated with minimal side effects, such as diarrhoea and headache, and is not contraindicated in pregnancy. ${ }^{5}$ Some clinical trials have shown that a single $3 \mathrm{~g}$ dose of fosfomycin is as effective as a 7-to-a-10-day regimen using other oral antibiotics to treat uncomplicated cystitis. ${ }^{5}$ The findings of a more recent open-label randomized trial suggest that fosfomycin efficacy for acute uncomplicated UTI in comparison to that of nitrofurantoin is lower. 
The oral formulation of fosfomycin has only recently become available at our centre. Susceptibility testing for fosfomycin on urinary isolates has since been introduced at Charlotte Maxeke Johannesburg Academic Hospital (CMJAH) microbiology laboratory. Consequently, there was a lack of information regarding the susceptibility rates of fosfomycin amongst uropathogens isolated at our centre. In this study, we aimed to analyse retrospective data for fosfomycin susceptibility of gram-negative and gram-positive bacteria isolated from urine samples and to compare its activity with that of other oral antimicrobials usually used to treat lower UTIs. Furthermore, the burden of MDR uropathogens was also established. This information will assist in determining the role of oral fosfomycin at our centre. In addition, it will help with the formulation of guidelines for therapy of lower UTIs locally.

\section{Methods Study design}

This is a retrospective laboratory-based study undertaken at CMJAH microbiology laboratory. Charlotte Maxeke Johannesburg Academic Hospital is a 1088-bed university hospital. Charlotte Maxeke Johannesburg Academic Hospital offers secondary, tertiary and quaternary services, as well as outpatient clinics. It also serves as a referral centre for several hospitals.

Antimicrobial susceptibility testing (AST) data for uropathogens isolated at CMJAH from September 2015 to August 2017 were used for analysis. Furthermore, the burden of MDR uropathogens for specific high-risk units for multidrug-resistant organisms (MDROs) was established. The data for this study were retrieved from the National Health Laboratory Service (NHLS) Corporate Data Warehouse (CDW).

\section{Definitions}

Multidrug resistance was defined as acquired non-susceptibility to at least one agent in three or more antimicrobial classes. ${ }^{8}$

AmpC- $\beta$-lactamase producers (Amp C): Organisms resistant to cefoxitin were considered to be Amp-C producers.

Extended-spectrum $\beta$-lactamases (ESBLs): Organisms resistant to ceftriaxone or ceftazidime were considered to be ESBL producers.

Carbapenem-resistant Enterobacteriaceae (CRE): Organisms resistant to ertapenem were considered to be carbapenem resistant.

\section{Sample size}

In total 8906 bacterial isolates were included in the study. Repeat bacteria cultured from the same patient were considered to be duplicate organisms representing a single infection if isolated within 2 weeks and were removed from the analysis. ${ }^{9}$ Fosfomycin results were not available for all the Enterobacteriaceae in the data retrieved from the CDW.
In addition, fosfomycin susceptibility testing of grampositive bacteria was performed on 76 selected non-duplicate isolates identified in the microbiology laboratory between June 2017 and August 2017.

\section{Bacterial culture and identification}

The microbiological diagnosis of a UTI requires $\geq 10^{5}$ colonyforming units $(\mathrm{CFU} / \mathrm{mL})$ of a single microorganism in a midstream collected urine, or $\geq 10^{4} \mathrm{CFU} / \mathrm{mL}$ of a single microorganism in catheter samples, or any bacterial growth in urine obtained by suprapubic route..$^{10}$ Bacterial culture and identification of urine specimens were performed according to the CMJAH Microbiology Laboratory Urine Standard Operating Procedures. Urine specimens were inoculated on $5 \%$ blood agar and MacConkey agar plates and incubated overnight at $35^{\circ} \mathrm{C}-37^{\circ} \mathrm{C}$. The bacterial species identification was performed using the $\mathrm{API}^{\circledR}$ 20E kit (bioMérieux SA, France) or the $\operatorname{Vitek}^{\circledR} 2$ system (bioMérieux SA, France).

\section{Antimicrobial susceptibility testing}

Routine AST of the isolates was performed using the disk diffusion method or the Vitek $^{\circledR} 2$ system (bioMérieux SA, France) according to the Clinical and Laboratory Standards Institute (CLSI). 11,12,13 Fosfomycin susceptibility was determined by the disk diffusion method. The plates were incubated aerobically at $35^{\circ} \mathrm{C}-37^{\circ} \mathrm{C}$ for $18-24 \mathrm{~h}$. The results were interpreted according to the CLSI guidelines breakpoints for the corresponding year. ${ }^{11,12,13}$ The CLSI provides fosfomycin interpretive breakpoints for Escherichia coli and Enterococcus faecalis only. The fosfomycin interpretive breakpoints for E. coli were used to interpret fosfomycin susceptibility for other Enterobacteriaceae, whilst the interpretive breakpoints for E. faecalis were used for grampositive bacteria. ${ }^{2}$ For Enterobacteriaceae, CLSI interpretative breakpoints for cefazolin were used to predict susceptibility to cephalexin. Two bacterial strains were used for quality control (QC) of AST: E. coli (American Type Culture Collection [ATCC] 25922) and Staphylococcus aureus (ATCC 25923). ${ }^{11,12,13}$ Quality control was performed weekly.

\section{Data analysis}

Fosfomycin and other oral antibiotic susceptibility results were reported as percentage susceptible. The difference between the percentage of isolates susceptible to fosfomycin and other oral antibiotics was calculated using 95\% confidence intervals (CIs) and $p$ values.

The 95\% CI was calculated using the Poisson distribution. A $p<0.05$ was considered statistically significant. All statistical analyses were conducted using STATA, version 14 (StataCorp, USA).

\section{Ethical consideration}

The study was accepted by the Human Research Ethics Committee (Medical) of the University of the Witwatersrand, approval number M170935. 


\section{Results}

\section{Distribution of isolated uropathogens from Charlotte Maxeke Johannesburg Academic Hospital}

The distribution of isolated uropathogens from CMJAH is shown in Table 1. From September 2015 to August 2017, a total of $9083(21.9 \%)$ urines were culture positive. The predominant causative organisms were E. coli, Klebsiella pneumoniae and E. faecalis.

\section{Fosfomycin susceptibility testing results}

Fosfomycin susceptibility testing results were available for 4700 Enterobacteriaceae. The overall susceptibility of fosfomycin for the Enterobacteriaceae was 95.7\%; 95\%

TABLE 1: Distribution of uropathogens isolates from Charlotte Maxeke Johannesburg Academic Hospital.

\begin{tabular}{lcc}
\hline Uropathogens & $\boldsymbol{N}$ & $\mathbf{\%}$ \\
\hline Total number of specimens $\dagger$ & $\mathbf{4 1 4 4 6}$ & - \\
\hline Total number of specimens with uropathogens & $\mathbf{9 0 8 3}$ & - \\
Total gram-negative bacilli isolated & $\mathbf{7 3 9 5}$ & $\mathbf{8 1 . 4}$ \\
E. coli & 4142 & 56.0 \\
Klebsiella pneumoniae & 1252 & 16.9 \\
Klebsiella oxytoca & 98 & 1.3 \\
Proteus mirabilis & 475 & 6.4 \\
Enterobacter species & 314 & 4.2 \\
Citrobacter species & 129 & 1.7 \\
Pseudomonas aeruginosa & 364 & 4.9 \\
Acinetobacter baumannii & 241 & 3.2 \\
Others & 380 & 5.1 \\
Total gram-positive cocci isolated & 1511 & $\mathbf{1 6 . 6}$ \\
S. aureus & 143 & 9.5 \\
S. saprophyticus & 12 & 0.8 \\
E. faecalis & 792 & 52.4 \\
E. faecium & 182 & 12.1 \\
S. agalactiae & 227 & 15.0 \\
Others & 155 & 10.3 \\
Total Candida species & $\mathbf{1 7 7}$ & $\mathbf{2 . 0}$ \\
Candida albicans & 97 & 54.8 \\
Non-albicans Candida species & 80 & 45.2 \\
\hline U & &
\end{tabular}

$\dagger$, Urine samples from September 2015 to August 2017 (study period).

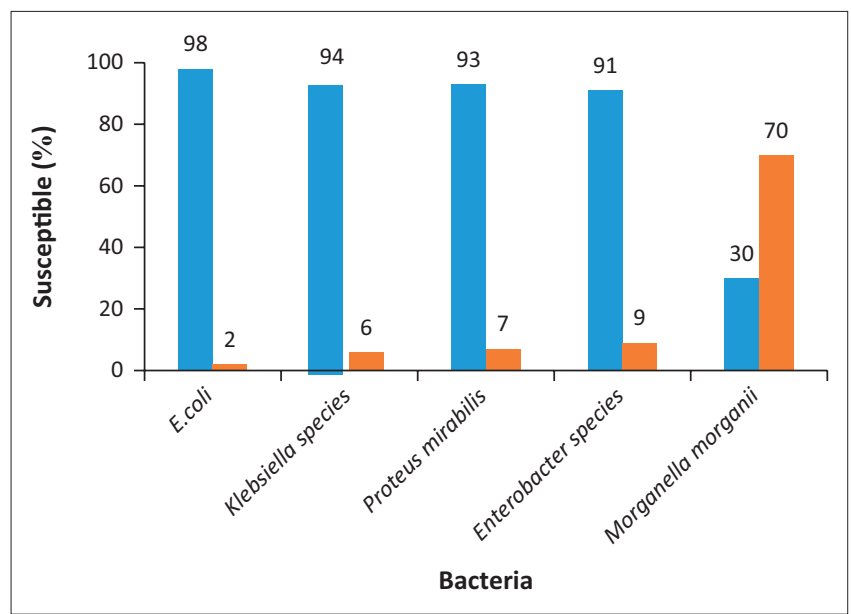

FIGURE 1: Antimicrobial susceptibility of urinary Enterobacteriaceae to fosfomycin.
CI: 95.1-96.2; $p$-value of 0.0001. Fosfomycin activity against E. coli was $98.1 \%$. Other members of the Enterobacteriaceae family in this current study also showed high susceptibility to fosfomycin except for Morganella species (Figure 1).

A breakdown of the 76 gram-positive organisms tested for fosfomycin susceptibility demonstrated 47 (61.8\%) E. faecalis, 15 (19.7\%) Enterococcus faecium, 7 (9.2\%) S. aureus, $4(5.3 \%)$ Streptococcus agalactiae and $3(3.9 \%)$ Staphylococcus saprophyticus isolates. As S. saprophyticus is intrinsically resistant to fosfomycin, these isolates were removed from the analysis. The overall susceptibility for fosfomycin for the gram-positives was 98.6\%. All the S. aureus isolates tested were susceptible to fosfomycin. Fosfomycin activity against enterococcal species, including the vancomycin-resistant enterococci (VRE) $(n=3)$ strains, was excellent. A single E. faecalis isolate was resistant to fosfomycin (Figure 2).

\section{Multidrug-resistant rates}

There were 2520 (37.9\%) multidrug-resistant Enterobacteriaceae (MDRE) isolated during the study period. Extendedspectrum $\beta$-lactamase production was identified in $23.2 \%$ $(n=1545)$ of the Enterobacteriaceae (Table 2). The majority of ESBL producers were K. pneumoniae $636(41.2 \%)$ and E. coli $=623(40.3 \%)$ (data not shown). Overall, $24(17 \%)$ of S. aureus isolates were methicillin-resistant Staphylococcus aureus (MRSA) and 11 (1.1\%) of the Enterococcus species isolates were VRE (data not shown). The majority of MDRE were isolated from patients admitted to ICUs (Figure 3).

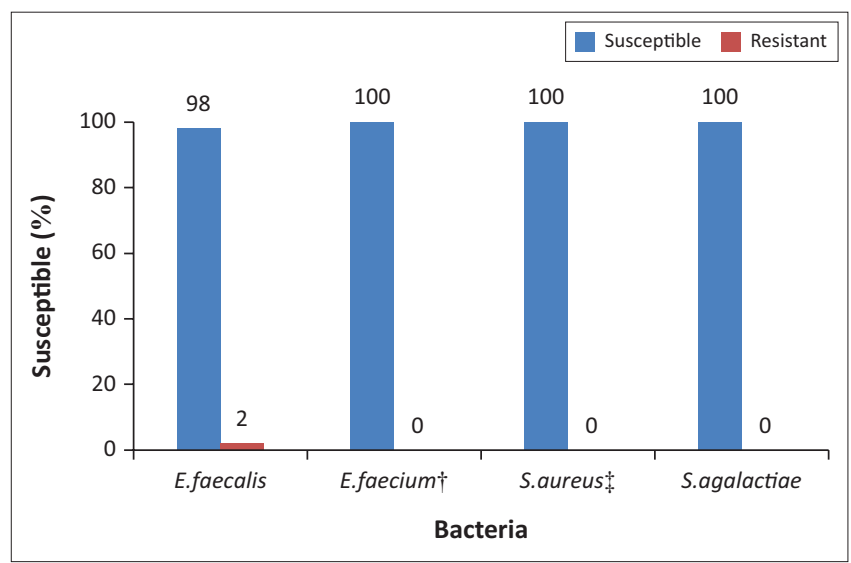

$\dagger$, Includes VRE; $\$$, Includes MRSA, methicillin-resistant Staphylococcus aureus. VRE, vancomycin-resistant enterococci.

FIGURE 2: Antimicrobial susceptibility of urinary gram-positive cocci to fosfomycin.

TABLE 2: Percentage of multidrug-resistant Enterobacteriaceae urinary isolates.

\begin{tabular}{lcc}
\hline MDRE & $\boldsymbol{N}$ & $\mathbf{\%}$ \\
\hline Total number of Enterobacteriaceae & 6652 & - \\
Total number of MDRE & 2520 & 37.9 \\
ESBL & 1545 & 23.2 \\
AmpC & 815 & 12.3 \\
CRE & 160 & 2.4 \\
\hline
\end{tabular}

MDRE, multidrug-resistant Enterobacteriaceae; AMP C, AmpC $\beta$-lactamase producers; ESBL, extended spectrum $\beta$-lactamase producers; CRE, carbapenem-resistant Enterobacteriaceae. 


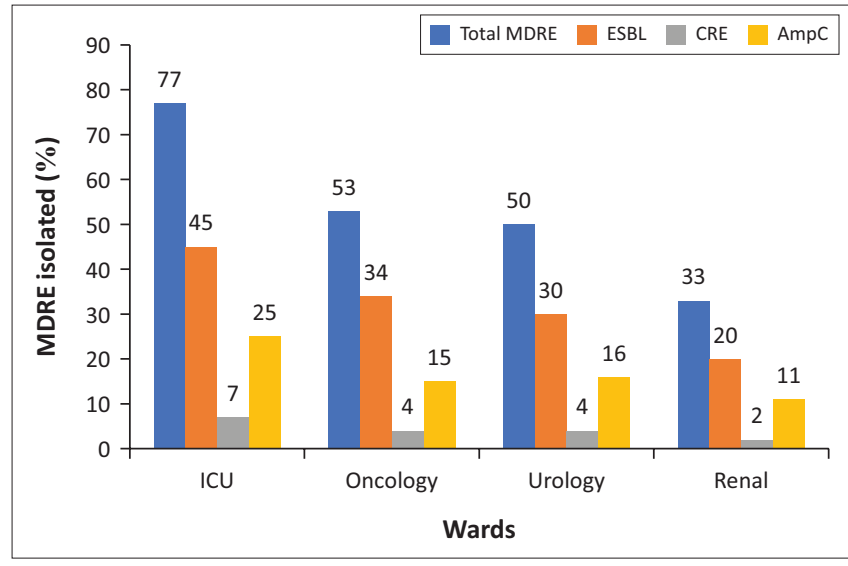

MDRO, multidrug-resistant organisms; ICU, intensive care unit; AMP C, AmpC $\beta$-lactamase producers; ESBL, extended spectrum $\beta$-lactamase producers; CRE, carbapenem-resistant Enterobacteriaceae.

FIGURE 3: Multidrug-resistant enterobacteriaceae urinary isolates at high risk units for MDRO.

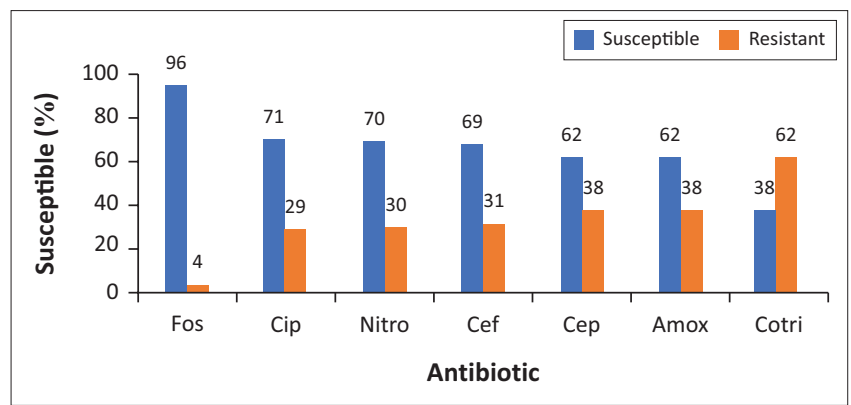

UTIs, urinary tract infections; Fos, fosfomycin; Cip, ciprofloxacin; Nitro, nitrofurantoin; Cef, cefuroxime; Cep, cephalexin; Amox, amoxicillin-clavulanate; Cotri, trimethoprim/ sulfamethoxazole; MDRE, multidrug-resistant Enterobacteriaceae.

FIGURE 4: Antimicrobial susceptibility of fosfomycin compared with other oral antibiotics used to treat Enterobacteriaceae causing urinary tract infections (including multidrug-resistant Enterobacteriaceae [MDRE]).

For the subset of MDRE with fosfomycin results available, the fosfomycin susceptibility was: $94.4 \%$ for ESBL producers and $90.7 \%$ for CRE. All the MRSA and VRE isolates tested showed 100\% susceptibility to fosfomycin (data not shown).

\section{Fosfomycin susceptibility compared with other oral agents used to treat Enterobacteriaceae causing urinary tract infections}

In the present study, fosfomycin showed the highest susceptibility (95.7\%, 95\% CI: 95.1-96.2) against the tested Enterobacteriaceae. There was a statistically significant difference between the susceptibility rates of fosfomycin $(p=0.0001)$ and all the other oral agents tested. Ciprofloxacin, cefuroxime and nitrofurantoin susceptibility rates were modest; however, they were much lower compared to that of fosfomycin. The lowest susceptibility was seen with trimethoprim/ sulfamethoxazole (38.35\%, 95\% CI: 37.2-39.5) (Figure 4).

\section{Discussion}

The key finding of this study is that fosfomycin shows outstanding in vitro activity against uropathogens, including MDR isolates. Overall, the susceptibility of fosfomycin against Enterobacteriaceae was $95.7 \%$. Fosfomycin activity against $E$. coli was higher than that of other Enterobacteriaceae. Significant susceptibility rates to fosfomycin were also found for K. pneumoniae, P. mirabilis and the tested gram-positive isolates. These findings are similar to those reported in the literature. ${ }^{4,14,15}$ Morganella species strains displayed significantly reduced susceptibility compared to other Enterobacteriaceae (70\% resistance rates). This is in keeping with other studies, where fosfomycin-resistant rates for Morganella morganii range between $75 \%$ and $100 \% .{ }^{16,17}$ M. morganii is regarded as being intrinsically resistant to fosfomycin. ${ }^{18}$ A study by Ito et al. ${ }^{19}$ detected fosA alleles in more than $90 \%$ of the M. morganii isolates. Production of fosfomycin-inactivating enzyme FosA is encoded by some clinically relevant gram-negative species and contributes to intrinsic fosfomycin resistance. ${ }^{19}$

The overall susceptibility of fosfomycin for gram-positives in this study was $98.6 \%$. The most frequent gram-positive uropathogens were Enterococcus species, which were highly susceptible (98\%) to fosfomycin. Research by Sultan et al. ${ }^{20}$ found that fosfomycin was highly effective against Enterococcus species and Staphylococcus species including VRE and MRSA. Fosfomycin is active against S. aureus biofilm production..$^{21}$ Bacterial biofilm can play an important role in recurrent UTIs and resistance to antimicrobial agents.

Fosfomycin also demonstrated good activity against UTIs caused by MDRO. In this current study, the susceptibility of fosfomycin for MDRE causing UTIs was 90.7\% (CRE), 92.3\% (AmpC) and $94.4 \%$ (ESBL). Comparable to this study, a systematic review of E. coli ESBL susceptibility to fosfomycin revealed susceptibility rates of more than $81 \% .^{22}$ All MRSA and VRE tested in this research were susceptible to fosfomycin. Because of the high fosfomycin susceptibility rates against uropathogens in our setting, fosfomycin is an attractive choice of treatment for outpatients and hospitalised patients with suspected or culture-confirmed MDR (MDRE, VRE and MRSA) UTIs. A significant pharmacological benefit of fosfomycin is that it can be administered orally. The use of fosfomycin could prevent admission for the treatment of MDR UTIs or decrease the length of hospital stay by allowing substitution of oral for intravenous therapy. Fosfomycin could also prove useful for the treatment of UTIs in circumstances where therapy with conventional antibiotics was unsuccessful or is contraindicated. Furthermore, the use of aminoglycosides may be retained for MDR A. baumannii and $P$. aeruginosa treatment.

Studies have demonstrated the clinical efficacy of fosfomycin as therapy for lower UTIs caused by both susceptible and MDR uropathogens. A report by Neuner confirmed microbiological cure rates in $71 \%$ of patients with UTIs caused by VRE and ESBL producers. The study population included patients with co-morbidities such as diabetes mellitus, solid-organ transplant recipients and chronic kidney disease. ${ }^{15}$ Another study by Nagel et al., ${ }^{23}$ compared clinical outcomes of fosfomycin in the hospital setting for patients with susceptible and MDR UTIs. A cure rate of $95 \%$ 
was documented. The majority of patients had UTIs caused by VRE $(45 \%) .{ }^{23}$ A cohort study of hospitalised patients treated with oral fosfomycin for uncomplicated or complicated UTIs showed favourable clinical outcomes, with cure rates of $75 \%-90 \%$ for the different cohorts studied. Their study included patients with comorbidities and an equal proportion of community-acquired and nosocomial infections in the cohorts studied. ${ }^{24}$ A multinational study comparing a 5-day course of nitrofurantoin to a single 3-g dose of fosfomycin for uncomplicated UTIs in non-pregnant females showed clinical and microbiological cure rates of $58 \%$ and $63 \%$, respectively, for the fosfomycin group..$^{19}$ These were both significantly lower than that of the nitrofurantointreated group. Based on the findings of this study, further well-designed clinical trials assessing the role of fosfomycin is required. ${ }^{25}$

Globally, the susceptibility rates of fosfomycin have continued to be quite stable since its introduction. Studies performed in three European countries, where parenteral and oral fosfomycin has been used since the 1970s, showed no significant decrease in fosfomycin susceptibility. ${ }^{26}$ Fosfomycin exhibits a low level of cross-resistance to other antimicrobial agents because of its unique mechanism of action..$^{14}$

Enterobacteriaceae are the common aetiological agents of UTIs. ${ }^{3,4}$ Our epidemiology is in keeping with the existing literature, where the majority of bacterial isolates were gramnegative bacilli $(81.4 \%)$. The predominant causative organisms in this current study were E. coli, K. pneumoniae and E. faecalis. Even though gram-negative bacteria are the main causative organisms for complicated and uncomplicated UTIs, gram-positive bacteria are also important uropathogens. ${ }^{3,4}$ S. saprophyticus, E. faecalis, and S. agalactiae are the common cause of community-acquired UTIs in pregnant women and the elderly. E. faecalis and E. faecium are the third leading cause of nosocomial UTIs. ${ }^{27}$ The ratio of E. faecalis to E. faecium in this current study was $4: 1$. The incidence of UTIs caused by E. faecalis has escalated gradually and now outnumbers E. faecium UTIs. ${ }^{27}$

Rates of both nosocomial and community-acquired UTIs caused by MDR bacteria are escalating. ${ }^{3,5,28}$ In this study, $37.9 \%$ of Enterobacteriaceae were MDR. The ESBL phenotype resistance pattern was the common MDR pattern detected. $\beta$-Lactamase producers can express resistance to other classes of antibiotics. ${ }^{29}$ The increasing rate of resistance to multiple antibiotic classes and the high rate of MDRE may lead clinicians to use more carbapenems and colistin. Colistin is a potentially nephrotoxic antibiotic and is associated with neurological toxicity. ${ }^{30}$ Carbapenems and colistin are parenteral antibiotics and necessitate patient hospitalisation, which is linked to substantial financial burdens. Systemic exposure to broad-spectrum antibiotics is also associated with increased selection of MDR pathogens. ${ }^{31}$ This study shows that MRSA (17\%) isolates are common uropathogens in our institution (data not shown). A comparable MRSA rate
(14\%) was reported in a study by Rajuduraipandi. ${ }^{32}$ In contrast, higher rates of $80.9 \%$ in Indore and $50.4 \%$ in eastern Uttar Pradesh were reported. ${ }^{33,34}$ This variability could be because of the difference in study design, population, antibiotic prescribing habits and geographic distribution.

This study did not look at the risk factors associated with MDRE. Rather, we focused on establishing the burden of MDRE uropathogens in selected wards. Previous studies have acknowledged that prior fluoroquinolone use within 3 months, a hospital stay for at least $48 \mathrm{~h}$, comorbidities, an indwelling urinary catheter, and urological procedures within the past 3 months are independent risk factors linked with MDRE UTIs. ${ }^{35}$ The distribution of MDRE UTIs in the selected high-risk wards is shown in Figure 3. The majority of MDRE UTIs were from patients admitted to ICU (77\%). Indwelling urinary catheters, the length of ICU admission and selective pressure from the use of antimicrobial agents are the most likely risk factors for the high rates of MDRE in ICU. The findings emphasise the significance of antibiotic resistance surveillance data and the knowledge of the risk factors associated with MDR UTIs in guiding empiric therapy choices.

The initiation of appropriate antimicrobial agents empirically is of utmost importance. However, in order to optimize empiric therapy, local patterns of antimicrobial resistance should be known. Antibiotic resistance rates and patterns differ by patient population type and change over time. ${ }^{36}$ The IDSA 2010 guidelines recommend a $10 \%$ to $20 \%$ resistance threshold for the treatment of community-acquired UTIs using a particular antibiotic; above this threshold, the agent should not be used for empiric therapy. ${ }^{6}$

In our setting, when compared with other oral agents used for UTIs, isolates were more frequently susceptible to fosfomycin. Fosfomycin susceptibility rates for Enterobacteriaceae were the highest, followed by ciprofloxacin, nitrofurantoin and cefuroxime. Our data confirmed decreased ciprofloxacin susceptibility amongst the Enterobacteriaceae tested. Although fluoroquinolones are highly efficacious in a 3-day regimen, there has been a dramatic increase in resistance to these agents in gramnegative uropathogens in the past decade. A multicentre study conducted between 2007 and 2011 detected a significant decrease in ciprofloxacin susceptibility amongst E. coli causing UTIs in both the public and the private sector. Ciprofloxacin susceptibility in the public sector declined by $5 \%$ and by $7 \%$ in the private sector. ${ }^{28}$ Ongoing indiscriminate and widespread use of ciprofloxacin to treat different infections is expected to cause a further decline in the susceptibility of pathogenic microorganisms to ciprofloxacin. This is particularly concerning, as both the South African Antibiotic Stewardship Programme Adult Antibiotic Guidelines and the Standard Treatment Guidelines and Essential Medicines List for South Africa (SA) recommend ciprofloxacin for management of cystitis and pyelonephritis in patients that are not severely ill and are able to tolerate oral 
medication. ${ }^{37,38}$ Empirical treatment with ciprofloxacin would be inadequate in almost $30 \%$ of patients with UTI at our centre. Therefore, the use of ciprofloxacin at our centre should be reserved for the treatment of UTIs caused by bacteria with known susceptibility to this drug.

Nitrofurantoin susceptibility (70.1\%) was lower compared to findings from previous studies in our region, where the reported susceptibility was above $90 \%{ }^{4,20}$ Nitrofurantoin has a reasonable efficacy and a low predisposition to cause collateral damage. ${ }^{4,28}$ Nitrofurantoin is an alternative antibiotic for the treatment of UTIs caused by MDRE, but it is contraindicated in advanced pregnancy and in patients with significant renal dysfunction. ${ }^{39}$

Even though the first-generation cephalosporins reach high concentrations in the urine, they have high resistance rates when compared with other agents. ${ }^{40} \mathrm{~A}$ study by Chen et al. found a significant decline over a 10-year period for the sensitivity of E. coli to first-generation cephalosporins. The sensitivity rate was $72 \%$ in 2003 and $28 \%$ in $2012 .{ }^{41}$ In 2011, CLSI modified cefazolin breakpoints and this likely contributed to the higher observed resistance rates in this current study. Therefore, this antibiotic cannot be used for empiric UTIs treatment in our institution. The susceptibility of cefuroxime in our study was lower $(61.9 \%)$ compared to that found by Lewis. ${ }^{4}$ Previous widespread use of cefuroxime for other indications may have contributed to the declined susceptibility.

Although trimethoprim/sulfamethoxazole is still widely used and recommended for acute cystitis therapy, the limitation is that local resistance rates of uropathogens should not exceed $20 \%$, or the infecting strain should be confirmed to be susceptible. ${ }^{6}$ In the present study, the rate of trimethoprim/ sulfamethoxazole susceptibility $(61.6 \%)$ was the lowest amongst the oral antimicrobials tested. A study conducted in South Africa's Gauteng province similarly found trimethoprim/sulfamethoxazole to be the least efficacious antimicrobial agent against uropathogens causing communityacquired UTIs. ${ }^{4}$ Trimethoprim/sulfamethoxazole has been used as a prophylactic agent against opportunistic infections in people with Acquired Immunodeficiency Syndrome (AIDS) in SA. This may be contributing to the particularly high rates of resistance. Trimethoprim/sulfamethoxazole must be reserved for the treatment of uncomplicated community acquired UTIs where susceptibility of the organism has been confirmed.

Fosfomycin has also been used off-label with success for complicated UTIs excluding pyelonephritis, perinephric abscess or bacteraemic UTIs. ${ }^{42}$ The doses that have been used for complicated UTIs and MDRE vary in literature, for example, 3 g every $48-72 \mathrm{~h}$ for 6-21 days has been used. These multi-dose off-label regimens require further microbiological and clinical validation..$^{43}$ Dose adjustment is not required in susceptible groups, such as pregnant women, patients of advanced age, and those with hepatic or renal failure. Therefore, it is a safe drug for patients with hepatic or renal dysfunction or for those receiving concomitant nephrotoxic drugs. ${ }^{5,44}$ Fosfomycin may be used in children; however, the availability of pharmacokinetic data for neonates is very scanty. The dose that has been used for children less than 5 years is $1 \mathrm{~g}-2 \mathrm{~g}$, but this dose strength is currently not available in SA. ${ }^{38}$

Gardiner et al..$^{45}$ found that the concentration attained by oral fosfomycin in the prostate is sufficient to prevent prostatic infection in patients undergoing transrectal ultrasoundguided biopsy. Fosfomycin may also be considered as an alternative treatment for prostatic infections caused by MDRE. ${ }^{45}$ The usual dosage regimen is $3 \mathrm{~g}$ of oral fosfomycin $3 \mathrm{~h}$ before and $24 \mathrm{~h}$ after traumatic interventions and surgical procedures. $^{46}$ Studies that compared the efficacy of ciprofloxacin versus fosfomycin when used for prophylaxis of urological procedures demonstrated that fosfomycin was associated with lower rates of subsequent UTIs. ${ }^{47,48}$

Research suggests that surgical antibiotic prophylaxis is beneficial for preventing UTIs post-urologic surgeries and procedures. ${ }^{46}$ The selection of the antibiotic used for surgical prophylaxis must also be based on the local epidemiology of antibiotic resistance. ${ }^{46}$ This study did not assess the role of fosfomycin in surgical prophylaxis for urological surgeries and procedures. However, based on the susceptibility of the microorganisms causing UTIs as shown in our research, fosfomycin could be used for surgical prophylaxis in our setting. It is vital to perform local studies to determine the role of fosfomycin above current antibiotic regimens used for surgical prophylaxis.

\section{Study limitations}

This study has several limitations. It was a retrospective single-centre study; therefore, the findings may not be generalised to other institutions with different susceptibility patterns and patient populations. A larger dataset, which includes data from other public and private hospitals, would offer further insight. As CMJAH is a referral hospital, antibiotic resistance rates may be higher than those found in local hospitals. Clinical and Laboratory Standards Institute breakpoints for E. coli and E. faecalis were extrapolated for the other Enterobacteriaceae and gram-positive organisms, respectively. Confirmatory tests for AmpC- $\beta$-lactamase and ESBL production were not performed. Molecular confirmation of carbapenemases production was not performed. Colistin broth microdilution was not performed in this study; therefore, we were not able to comment on the rate of colistin resistance. The small sample size of grampositives may limit the ability to make reliable conclusions about the activity of fosfomycin against these uropathogens in our setting. This needs to be addressed by performing a larger evaluation. This study was not designed to differentiate between community-acquired and nosocomial UTIs. Lastly, the study focused only on the in vitro susceptibility rates of uropathogens, and was not designed to assess clinical and microbiological outcomes. 


\section{Conclusion}

This study is important in that it represents the first local comprehensive evaluation of fosfomycin against gramnegative and gram-positive uropathogens. Fosfomycin has been shown to have good activity against both susceptible and MDR uropathogens. These findings together with its favourable side-effect profile, position it as a potential alternative oral agent for empiric and definitive treatment of UTIs for hospitalised and ambulatory patients at our centre.

\section{Acknowledgement}

The authors would like express their gratitude to the microbiology laboratory staff of the CMJAH for their assistance.

\section{Competing interests}

The authors confirm that there are no known conflicts of interest associated with this publication and there has been no significant financial support for this work that could have influenced its outcome.

\section{Authors' contributions}

All authors, L.M.M., N.N.B. and T.N., have made a significant contribution to the work and have seen and approved the content. L.M.M. was responsible for the following: conception and design of the project; acquisition, analysis and interpretation of data; L.M.M. drafted and wrote the manuscript. N.N.B. and T.N. assisted with design of the project and data interpretation. L.M.M., N.N.B. and T.N. revised the manuscript for intellectual content and approved the final version to be published.

\section{Funding information}

This work was supported by the Department of Clinical Microbiology and Infectious Diseases, Witwatersrand University. Funding for this study was supported by research funds allocated by the University of Witwatersrand to the Department of Clinical Microbiology and Infectious Diseases.

\section{Data availability statement}

Data sharing is not applicable to this article as no new data were created or analysed in this study.

\section{Disclaimer}

The views and opinions expressed in this article are those of the authors and do not necessarily reflect the official policy or position of any affiliated agency of the authors.

\section{References}

1. Najar MS, Saldanha CL, Banday KA. Approach to urinary tract infections. Indian Nephrol. 2009 Oct;19(4):129-139. https://doi.org/10.4103/0971-4065.59333

2. Maraki S, Samonis G, Rafailidis PI, Vouloumanou EK, Mavromanolakis E, Falagas ME. Susceptibility of urinary tract bacteria to fosfomycin. Antimicrob Agents Chemother. 2009;53(10):4508-4510. https://doi.org/10.1128/AAC.00721-09
3. Habte TM, Dube S, Ismail N, Hoosen AA. Hospital and community isolates of uropathogens at a tertiary hospital in South Africa. S Afr Med J. 2009 Aug;99(8):584-587.

4. Lewis DA, Gumede LYE, Van der Hoven LA, et al. Antimicrobial susceptibility of organisms causing community-acquired urinary tract infections in Gauteng Province, South Africa. S Afr Med J. 2013;103(6):377-381. https://doi. org/10.7196/SAMJ.6722

5. Michalopoulos AS, Livaditis IG, Gougoutas V. The revival of fosfomycin. Int J Infect Dis. 2011 Nov;15(11):e732-e739. https://doi.org/10.1016/j.ijid.2011.07.007

6. Gupta K, Hooton TM, Naber KG, et al. International clinical practice guidelines for the treatment of acute uncomplicated cystitis and pyelonephritis in women: $A$ 2010 update by the Infectious Diseases Society of America and the European Society for Microbiology and Infectious Diseases. Clin Infect Dis. 2011 Mar 1;52(5):e103-e120. https://doi.org/10.1093/cid/ciq257

7. Huttner A, Kowalczyk A, Turjeman A, et al. Effect of 5-day nitrofurantoin vs singledose fosfomycin on clinical resolution of uncomplicated lower urinary tract infection in women. A randomized clinical trial. JAMA. 2018;319(17):1781-1789. https://doi.org/10.1001/jama.2018.3627

8. Magiorakos AP, Srinivasan A, Carey RB, et al. Multidrug-resistant, extensively drug-resistant and pandrug-resistant bacteria: An international expert proposal for interim standard definitions for acquired resistance. Clin Microbiol Infect. 2012 Mar;18(3):268-281. https://doi.org/10.1111/j.1469-0691.2011.03570.x

9. Centers for Disease Control. National healthcare safety network surveillance (NHSN) patient safety component manual [homepage on the Internet]. 2020 [cited 2020 Jul 21]. Available from: https://www.cdc.gov/nhsn/pdfs/pscmanual/ pcsmanual_current.pdf

10. Graham JC, Galloway A. ACP best practice no 167: The laboratory diagnosis of urinary tract infection. J Clin Pathol. BMJ. 2001 Dec;54(12):911-919. https://doi. org/10.1111/j.1469-0691.2011.03570.x

11. M100S25 | Performance standards for antimicrobial susceptibility testing [homepage on the Internet]. [cited 2017 Oct 10]. Available from: https://clsi.org/ standards/products/microbiology/documents/m100/

12. M100S26 | Performance standards for antimicrobial susceptibility testing [homepage on the Internet]. [cited 2017 Oct 10]. Available from: https://clsi.org/ standards/products/microbiology/documents/m100/

13. M100S27 | Performance standards for antimicrobial susceptibility testing [homepage on the Internet]. [cited 2017 Oct 10]. Available from: https://clsi.org/ standards/products/microbiology/documents/m100/

14. Falagas ME, Roussos N, Gkegkes ID, Rafailidis PI, Karageorgopoulos DE. Fosfomycin for the treatment of infections caused by Gram-positive cocci with advanced antimicrobial drug resistance: A review of microbiological, animal and clinical studies. Exp Opin Investig Drugs. 2009;18(7):921-944. https://doi. org/10.1517/13543780902967624

15. Neuner EA, Sekeres J, Hall GS, Van Duin D. Experience with fosfomycin for treatment of urinary tract infections due to multidrug-resistant organisms. Antimicrob Ag

16. Fajfr M, Louda M, Paterová $P$, et al. The susceptibility to fosfomycin of Gram negative bacteria isolates from urinary tract infection in the Czech Republic: Data negative bacteria isolates from urinary tract infection in the Czech Republic: Data
from a unicentric study. BMC Urol. 2017 Apr 26;17(1):33. https://doi.org/10.1186/ s12894-017-0222-6

17. Demir T, Buyukguclu T. Evaluation of the in vitro activity of fosfomycin tromethamine against Gram-negative bacterial strains recovered from community- and hospital-acquired urinary tract infections in Turkey. Int J Infect Dis. 2013 Nov;17(11):e966-e970. https://doi.org/10.1016/j.ijid.2013.04.005

18. Stock I, Wiedemann B. Identification and natural antibiotic susceptibility of Morganella morganii. Diagn Microbiol Infect Dis. 1998;30(3):153-165. https:// doi.org/10.1016/S0732-8893(97)00243-5

19. Ito $R$, Mustapha $M M$, Tomich $A D$, et al. Widespread fosfomycin resistance in Gram- negative bacteria attributable to the chromosomal fosA gene. mBio. 2017;8:e00749-17. https://doi.org/10.1128/mBio.00749-17

20. Sultan A, Rizvi M, Khan F, Sami $H$, Shukla I, Khan HM. Increasing antimicrobial resistance among uropathogens: Is fosfomycin the answer? Urol Ann 2015;7(1):26-30. https://doi.org/10.4103/0974-7796.148585

21. Shen F, Tang $X$, Cheng $W$, et al. Fosfomycin enhances phagocyte-mediated killing of Staphylococcus aureus by extracellular traps and reactive oxygen species. Sci Rep. 2016 Jan 18;6(1):19262. https://doi.org/10.1038/srep19262

22. Vardakas KZ, Legakis NJ, Triarides N, Falagas ME. Susceptibility of contemporary isolates to fosfomycin: A systematic review of the literature. Int J Antimicrob Agents. isolates to fosfomycin: A systematic review of the literature. Int J Antimicrob Age
$2016 \mathrm{Apr} ; 47(4): 269-285$. https://doi.org/10.1016/j.ijantimicag.2016.02.001

23. Nagel JL, Washer L, Kunapuli A, Heidmann J, Pisani J, Gandhi T. Clinical efficacy of fosfomycin for the treatment of complicated lower tract and uncomplicated urinary tract infections. Int Arch Med. 2015;8(151):1-7. https://doi.org/10.3823/1750

24. Sastry S, Clarke LG, Alrowais H, Querry AM, Shutt KA, Doi Y. Clinical appraisal of fosfomycin in the era of antimicrobial resistance. Antimicrob Agents Chemother. 2015 Dec;59(12):7355-7361. https://doi.org/10.1128/AAC.01071-15

25. Datta R, Juthani-Mehta $M$. Nitrofurantoin vs Fosfomycin rendering a verdict in a trial of acute uncomplicated cystitis. JAMA. 2018;319(17):1771-1772. https://doi org/10.1001/jama.2018.4654

26. Karageorgopoulos DE, Wang R, Yu X-h, Falagas ME. Fosfomycin: Evaluation of the published evidence on the emergence of antimicrobial resistance in Gramnegative pathogens. J Antimicrob Chemother. 2012 Feb;67(2):255-268. https:// doi.org/10.1093/jac/dkr466

27. Kline KA, Lewis AL. Gram-positive uropathogens, polymicrobial urinary tract infection, and the emerging microbiota of the urinary tract. Microbiol Spect 2016 Jun 1;4(2), 1-31. https://doi.org/10.1128/microbiolspec.UTI-0012-2012 
28. Bamford C, Bonorchis K, Ryan A, et al. Antimicrobial susceptibility patterns of Escherichia coli strains isolated from urine samples in South Africa from 2007-2011. S Afr J Epidemiol Infect. 2012;27(2):46-52. https://doi.org/10.1080 2007-2011. S Afr J Epidemiol

29. Paterson DL, Bonomo RA. Extended-spectrum $\beta$-lactamases: A clinical update. Clin Microbiol Rev. 2005;18(4):657-686. https://doi.org/10.1128/CMR.18.4.657686.2005

30. Spapen H, Jacobs R, Van Gorp V, Troubleyn J, Honoré PM. Renal and neurological side effects of colistin in critically ill patients. Ann Intens Care. 2011 May 25;1(1):14. https://doi.org/10.1186/2110-5820-1-14

31. Brink A, Coetzee J, Clay C, et al. The spread of carbapenem-resistant Enterobacteriaceae in South Africa: Risk factors for acquisition and prevention. S Afr Med J. 2012 May 10;102(7):599-601. https://doi.org/10.7196/SAMJ.5789

32. Rajaduraipandi K, Mani KR, Panneerselvam, Mani M, Bhaskar M, Manikandan P. Prevalence and antimicrobial susceptibility pattern of methicillin resistant Staphylococcus aureus: A multicenter study. Indian J Med Microb. 2006 Staphylococcus aureus: A multicenter study. Indian J
Jan;24(1):34-38. https://doi.org/10.4103/0255-0857.19892

33. Verma S, Joshi S, Chitnis V, Hemwani N, Chitnis D. Growing problem of methicillin resistant Staphylococci: Indian scenario. Indian J Med Sci. 2000 Dec;54:535-540.

34. Anupurba S, Sen MR, Nath G, Sharma BM, Gulati AK, Mohapatra TM. Prevalence of methicillin resistant Staphylococcus aureus in a tertiary referral hospital in eastern Uttar Pradesh. Indian J Med Microbiol. 2003 Jan-Mar;21(1):49-51.

35. Khawcharoenporn T, Vasoo S, Singh K. Urinary tract infections due to multidrugresistant Enterobacteriaceae: Prevalence and risk factors in a Chicago emergency department. Emerg Med Int. 2013 Oct 31;2013:1-7. https://doi.org/10.1155/ 2013/258517

36. Abbo LM, Hooton TM. Antimicrobial stewardship and urinary tract infections. Antibiotics. 2014 May 5;3(2):174-192. https://doi.org/10.3390/antibiotics3020174

37. Wasserman S, Boyles T, Mendelson M. A pocket guide to antibiotic prescribing for adults in South Africa [homepage on the Internet]. 2015 [cited 2017 Oct 20]. Available from: http://www.fidssa.co.za/images/SAASP_Antibiotic_Guidelines_2015.pdf

38. Republic of South Africa. Essential Drugs Programme. Hospital level (adults) standard treatment guidelines and essential medicines list [serial online]. 4th ed. National Department of Health; 2015 [cited 2017 Oct 20]. Available from: http:// www.health.gov.za/edp.php
39. Matthews PC, Barrett LK, Warren S, et al. Oral fosfomycin for treatment of urinary tract infection: A retrospective cohort study. BMC Infect Dis, 2016 Oct 11;16(1):556. https://doi.org/10.1186/s12879-016-1888-1

40. Sanchez GV, Babiker A, Master RN, Luu T, Mathur A, Bordon J. Antibiotic resistance among urinary isolates from female outpatients in the United States in 2003 and 2012. Antimicrob Agents Chemother. 2016 Apr 22:60(5): 2680-2683. https://doi. org/10.1128/AAC.02897-15

41. Chen P-C, Chang L-Y, Lu C-Y, et al. Drug susceptibility and treatment response of common urinary tract infection pathogens in children. J Microbiol Immunol Infect. 2014;47(6):478-483. https://doi.org/10.1016/j.jmii.2013.07.011

42. Pallet $A$, Hand K. Complicated urinary tract infections: Practical solutions for the treatment of multiresistant Gram-negative bacteria. J Antimicrob Chemother. 2010 Nov;65(Suppl 3):iii25-iii33. https://doi.org/10.1093/jac/dkq298

43. Abbott IJ, Van Gorp E, Wijma RA, et al. Oral fosfomycin efficacy with variable urinary exposures following single and multiple doses against Enterobacterales: The importance of heteroresistance for growth outcome. Antimicrob Agents Chemother. 2020;64(Suppl 3):e01982-19. https://doi.org/10.1128/AAC 01982-19

44. Falagas ME, Vouloumanou EK, Samonis G, Vardakas KZ. Fosfomycin. Clin Microbiol Rev. 2016 Apr;29(2):321-347. https://doi.org/10.1128/CMR.00068-15

45. Gardiner BJ, Mahony AA, Ellis AG, et al. Is fosfomycin a potential alternative for multidrug-resistant gram-negative prostatitis? Clin Infect Dis. 2014;58(4) e101-e105. https://doi.org/10.1093/cid/cit704

46. Mrkobrada M, Ying I, Mokrycke $S$, et al. CUA guidelines on antibiotic prophylaxis for urologic procedures. Can Urol Assoc J. 2015 Jan-Feb;9(1-2):13-22. https:// doi.org/10.5489/cuaj.2382

47. Sen V, Aydogdu O, Bozkurt IH, et al. The use of prophylactic single-dose fosfomycin in patients who undergo transrectal ultrasound-guided prostate biopsy: A prospective, randomized, and controlled clinical study. Can Urol Assoc J. 2015;9(11-12):E863-E837. https://doi.org/10.5489/cuaj.3068

48. Cai T, Gallelli L, Cocci A, et al. Antimicrobial prophylaxis for transrectal ultrasound-guided prostate biopsy: Fosfomycin trometamol, an attractive alternative. World J Urol. 2017 Feb;35(2):221-228. https://doi.org/10.1007/ s00345-016-1867-6 